\title{
ACOLHIMENTO ÀS PESSOAS EM SOFRIMENTO PSÍQUICO: DESAFIO À REFORMA PSIQUIÁTRICA
}

EMBRACING PEOPLE WHO SUFFER FROM PSYCHOLOGICAL STRESS:

CHALLENGE TO THE PSYCHIATRIC REFORM

ACOGIMIENTO A LAS PERSONAS QUE SUFREN DE ESTRÉS PSICOLÓGICO: DESAFÍO A LA REFORMA PSIQUIÁTRICA

Palavras-chave: Reforma Psiquiátrica; Sofrimento Psíquico; Acolhimento; Psicologia.

Keywords:

Psychiatric Reform; Psychological Stress; User Embracement; Psychology.

Palabras clave: Reforma Psiquiátrica; Estrés Psicológico; Acogimiento; Psicología.

Submetido: $14 / 12 / 2017$

Aprovado: 02/05/2018

\section{Autor(a) para Correspondência:} Karolyne Braga Moreira End: Av. Santo Antônio, 591, Centro, Reriutaba-CE, CEP 62260-000 E-mail:karolbragam@hotmail.com

\section{RESUMO}

o modo como o profissional que atua nos equipamentos substitutivos da Reforma Psiquiátrica brasileira acolhe os sujeitos que apresentam ou descrevem alguma queixa de sofrimento psíquico representa um desafio. Assim, esta pesquisa buscou levantar em que medida as atuais práticas cotidianas na relação entre profissionais da saúde e usuários dos serviços de saúde se aproximam ou se afastam do ideal proposto pela Reforma Psiquiátrica no Brasil. Os acontecimentos políticos e sociais dos movimentos da Reforma Sanitária e da Reforma Psiquiátrica são retomados, para configurar o modo como o sujeito em sofrimento psíquico é acolhido atualmente nos serviços substitutivos da Reforma Psiquiátrica. Trata-se de pesquisa bibliométrica, com base em 29 artigos selecionados - que, em sua maioria, revelam os desafios e os impasses nos serviços de saúde, como: dificuldade de adotar práticas diferentes; não participação da categoria médica; desafios da implantação do apoio matricial; precarização das condições de trabalho; e não efetivação de ações cotidianas embasadas nas diretrizes propostas pelo Sistema Único de Saúde (SUS). As práticas observadas revelam que o Brasil ainda carece de uma Reforma Psiquiátrica de fato.

\footnotetext{
1. Graduada em Psicologia pela Universidade Federal do Ceará (UFC). Reriutaba (CE), Brasil. E-mail: karolbragam@hotmail.com

2. Professora na UFC. Meste em Psicologia e Doutora em Saúde Coletiva pela UFC. Pós-Doutora em Psicanálise pela Universidade do Estado do Rio de Janeiro (UERJ). Sobral (CE), Brasil. E-mail: tgd.camilla@gmail.com
} 


\section{ABSTRACT}

The way how a professional who works in the substitutive equipment of the Brazilian Psychiatric Reform embraces the subjects presenting or describing some complaint of psychological stress represents a challenge. Thus, this research sought to survey the extent to which current daily practices in the relation between health professionals and users of health services have been coming closer to or detaching from the ideal as proposed by the Psychiatric Reform in Brazil. The political and social events of the Health Care Reform and the Psychiatric Reform are resumed in order to set up the way how a subject suffering from psychological stress is currently embraced in the substitutive services of the Psychiatric Reform. This is a bibliometric survey, based on 29 selected articles - which, for the most part, reveal the challenges and deadlocks in health services, such as: difficulty in adopting different practices; no participation of the medical category; challenges to deploy the matrix support; precariousness of working conditions; and non-implementation of daily actions based on the guidelines proposed by the Brazilian National Health System (SUS). The practices observed reveal that Brazil still lacks a de facto Psychiatric Reform.

\section{RESUMEN}

La forma en que un profesional que trabaja en el equipo sustitutivo de la Reforma Psiquiátrica brasileña acoge a los sujetos que presentan o describen alguna queja de estrés psicológico representa un desafío. Así, esta investigación buscó encuestar hasta qué punto las actuales prácticas cotidianas en la relación entre profesionales de salud y usuarios de servicios de salud se han acercado o separado del ideal propuesto por la Reforma Psiquiátrica en Brasil. Los eventos políticos y sociales de la Reforma de la Atención de Salud y la Reforma Psiquiátrica se reanudan con el fin de establecer la forma en que un sujeto que sufre de estrés psicológico se acoge actualmente en los servicios sustitutivos de la Reforma Psiquiátrica. Esta es una encuesta bibliométrica, basada en 29 artículos seleccionados, que, en su mayoría, revelan desafios e impasses en los servicios de salud, como: dificultad para adoptar prácticas diferentes; no participación de la categoría médica; desafios para implementar el apoyo matricial; precariedad de las condiciones de trabajo; y no implementación de acciones cotidianas basadas en las directrices propuestas por el Sistema Único de Salud brasileño (SUS). Las prácticas observadas revelan que Brasil todavía carece de una Reforma Psiquiátrica de facto.

\section{INTRODUÇÃO}

0 modo como o profissional que atua nos equipamentos substitutivos da Reforma Psiquiátrica brasileira acolhe os sujeitos que apresentam ou descrevem alguma queixa de sofrimento psíquico representa um desafio às diretrizes em questão, tendo em vista que, muitas vezes, as práticas cotidianas na relação entre profissionais da saúde e usuários dos serviços de saúde fogem aos princípios dessa reforma.

Para falar sobre os principais aspectos que resultaram na criação do Sistema Único de Saúde (SUS), estabelecido pela Constituição Federal de 1988, deve-se compreender o contexto anterior à Reforma Sanitária. Em 1964, o Brasil passava pelo Golpe Militar, caracterizado pela desarticulação da participação social e pela busca de destruir todas as iniciativas de movimentos socialistas. No que diz respeito ao setor saúde, os governos militares buscaram incentivar a expansão da iniciativa privada.

As ideias do psiquiatra italiano Franco Basaglia (1924-1980) chegaram ao Brasil no final da década de 1970, acompanhando uma tendência internacional de mudanças para a superação do modelo asilar.
Desde então, as instituições de saúde foram identificando-se com tais mudanças e passaram a desenvolver ações com o objetivo de melhorar 0 atendimento nesse sentido. Na década de 1990, 0 país assinou a Declaração de Caracas, firmando seu compromisso com a implantação dos serviços embasados nos ideais da Reforma Sanitária ${ }^{1}$.

A partir disso, o Ministério da Saúde criou políticas e diretrizes para o funcionamento dos serviços substitutivos da Reforma Psiquiátrica com base nas conferências nacionais de saúde mental, que representam um processo político fundamental para mudanças em prol da melhoria nos serviços de saúde.

A I Conferência Nacional de Saúde Mental, de junho de 1987, apontou que as ações e os serviços de saúde deveriam formar o SUS, gratuito, descentralizado, regionalizado e hierarquizado. Dentre as principais novidades assistenciais havia a reversão da tendência "hospitalocêntrica e psiquiatrocêntrica", atribuindo prioridade ao sistema extra-hospitalar e multiprofissional como referência para o paciente, inserindo-se na estratégia de desospitalização. Também se determinou que o setor público não instalaria novos leitos psiquiátricos em 
unidades psiquiátricas hospitalares tradicionais, reduzindo progressivamente o número de tais leitos e substituindo-os por leitos psiquiátricos em hospitais gerais públicos ou por serviços inovadores alternativos à internação psiquiátrica. 0 acolhimento aos usuários de serviços de saúde mental deveria implicar a implantação de equipes multiprofissionais, de modo a reverter 0 modelo assistencial organicista e medicalizante ${ }^{2}$.

$\mathrm{Na}$ II Conferência Nacional de Saúde Mental, determinou-se a necessidade de estimular a criação de grupos de reflexão nos quais os profissionais de saúde mental possam repensar suas práticas ${ }^{3}$. De uma conferência para a outra foram propostas novas formas de acolher os sujeitos em sofrimento psíquico. Por exemplo, passou a se indicar que, em situações de crise, as intervenções devem ser realizadas em liberdade, recompondo os laços sociais do sujeito em sofrimento psíquico, tendo em vista a possibilidade do cuidado sem uso de violência, exclusão ou negação.

Nesse sentido, as reorientações do modelo assistencial propostas na III Conferência de Saúde Mental baseiam-se no lema “Cuidar, sim. Excluir, não: efetivando a Reforma Psiquiátrica, com Acesso, Qualidade, Humanização e Controle Social"4:11; houve significativo aumento do investimento em redes de atenção psicossocial substitutivas ao modelo "hospitalocêntrico" como eixo assistencial ${ }^{4}$.

$\mathrm{Na}$ IV Conferência Nacional de Saúde Mental, em 2010, pela primeira vez um evento intersetorial, houve grande participação de usuários, trabalhadores e gestores do setor saúde e de outros campos, a área da saúde mental passou a englobar direitos humanos, assistência social, educação, justiça, trabalho e economia solidária, habitação, cultura, lazer e esportes etc ${ }^{5}$. A partir dessa conferência, adotou-se, ainda, um modo menos estigmatizante de designar os usuários de serviços de saúde mental - surgiu a nomenclatura "pessoa em sofrimento psíquico"5:144, que distancia a concepção de portadora de alguma doença, antes atrelada aos usuários de tais serviços.

Em suas diretrizes, as conferências nacionais de saúde mental buscaram a desinstitucionalização, a inclusão social e a implantação de uma rede de assistência pautada no acolhimento dos sujeitos em sofrimento psíquico - que, segundo Emerson Merhy, deve ocorrer por meio de tecnologias leves de cuidado, um acolhimento pautado por um processo relacional, "mediante a escuta, o interesse, a construção de vínculos, de confiança; é [o] que

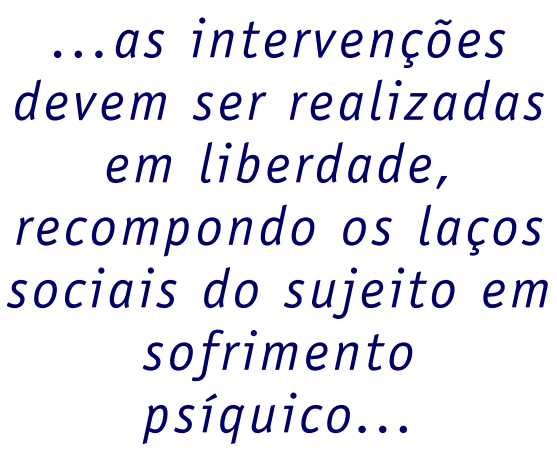

possibilita mais precisamente captar a singularidade, o contexto, o universo cultural"6:6.

Assim, esta pesquisa buscou levantar em que medida as atuais práticas cotidianas na relação entre profissionais da saúde e usuários dos serviços de saúde se aproximam ou se afastam do ideal proposto pela Reforma Psiquiátrica no Brasil.

\section{METODOLOGIA}

Trata-se de pesquisa bibliométrica baseada em artigos selecionados, isto é, textos científicos que trazem as informações relevantes diante dos objetivos em foco. Toda especialidade conta com determinado número de periódicos científicos onde se supõe encontrar os artigos mais importantes sobre os assuntos essenciais; a bibliometria proporciona meios para identificar tais periódicos, também denominados "nucleares", partindo da prática estabelecida na comunidade científica de informar as referências bibliográficas consultadas durante a elaboração de qualquer estudo ${ }^{7}$.

Por meio da pesquisa bibliométrica de trabalhos sobre o tema publicados no período de 2007 a 2017, foram elencadas as principais formas de atuação dos profissionais nos serviços substitutivos da Reforma Psiquiátrica. Identificou-se o que tem sido feito e de que modo o sujeito em sofrimento psíquico tem sido acolhido. Nessa perspectiva, adotou-se um recorte atualizado sobre tais questões, considerando que uma década constitui período de tempo significativo para levantar as publicações importantes acerca do tema.

$0 s$ artigos foram selecionados nas bases da Scientific Electronic Library Online (SciELO) e do Centro Latino-Americano e do Caribe de Informação em Ciências da Saúde (Bireme) - que reúnem periódicos científicos de qualidade de modo acessível e organizado, constituindo fontes de credibilidade em termos de produção científica.

Os critérios de inclusão dos artigos partiram das 
buscas por meio dos descritores em ciências da saúde (DeCS) sofrimento psíquico, acolhimento e reforma psiquiátrica. Como a palavra-chave crise advém, no setor saúde, do contexto das doenças orgânicas, constitui um emaranhado de possibilidades conceituais; assim, o DeCS sofrimento psíquico se mostrou mais efetivo em buscas específicas do que crise e, neste estudo, sujeito em crise se refere a sujeito em sofrimento psíquico. 0 DeCS acolhimento foi de suma importância para a seleção dos artigos, visto que o referencial teórico adotado o indica como vital para a atuação dos profissionais da saúde nos equipamentos substitutivos da Reforma Psiquiátrica. Por fim, o DeCS reforma psiquiátrica é indispensável nesse contexto, pois as novas formas de acolher os sujeitos em sofrimento psíquico se enquadram nas diretrizes propostas a partir dessa reforma.

Após a seleção dos artigos com base nos 3 DeCS indicados, eles foram submetidos a análise bibliométrica de títulos e resumos, para identificação das principais temáticas abordadas.

\section{RESULTADOS E DISCUSSÃO}

Os critérios de inclusão levaram à identificação de 57 artigos no total. Após a análise bibliométrica, foram selecionados 29 artigos para embasar a discussão dos paradigmas de funcionamento dos serviços substitutivos da Reforma Psiquiátrica. Todos esses artigos foram lidos na íntegra, com a finalidade de extrair as informações mais relevantes diante dos objetivos em foco.

Os dispositivos de saúde mais citados foram: a) centros de atenção psicossocial (Caps) - 12 artigos; b) serviços de atendimento básico - como Unidades Básicas de Saúde (UBS), Unidades de Atenção Primária à Saúde (APS), Unidades de Acolhimento (UA) e unidades de saúde mista - juntos, somam 8 artigos no total; e c) os outros serviços se referem a políticas do trabalhador (2 artigos), ambulatório de saúde mental (1 artigo) e hospital psiquiátrico (1 artigo).

A região onde mais se publicou sobre o assunto 0 Sudeste (15 artigos), seguida pelo Nordeste (7 artigos), pelo Sul (6 artigos) e pelo Centro-0este (1 artigo). Nesse sentido, percebe-se que o Sudeste concentra maior investimento em publicações, não significando necessariamente que em outras regiões se trabalhe menos.

No que se refere à relação entre as práticas e os ideais que norteiam a Reforma Psiquiátrica, 10 das publicações se referem a serviços cujos princípios são

\section{...entre os principais desafios estão as questões relacionadas ao acolhimento dos sujeitos...}

- acolhimento, a escuta, a integralidade do atendimento, a organização de uma rede de saúde mental, o apoio matricial, a intersetorialidade, a interdisciplinaridade, a desinstitucionalização e a promoção da cidadania - o que favorece a autonomia dos usuários. Nesse sentido, percebe-se que o universo total das publicações não faz referência a tais conceitos.

0 s artigos que se referem às práticas vivenciadas nos Caps parecem estar mais articulados aos princípios da Reforma Psiquiátrica brasileira. Pode-se citar como exemplo a experiência relatada em 2 artigos com construção de novos modos de fazer no âmbito da saúde mental. Um deles relata uma experiência em um Caps de São Paulo (SP), com a criação de um grupo de contação de histórias, criando espaços de escuta, acolhimento, lembranças e ressignificação de sentidos ${ }^{8}$. A outra experiência, em um Caps de Maceió $(A L)$, envolve a execução de oficinas com uso das Tecnologias da Informação e Comunicação (TIC); a partir da criação de um blog, a pessoa em sofrimento psíquico pode se expressar, compartilhar seus saberes, integrar-se em uma comunidade, ser reconhecida ao expor sua criatividade, dentre outras possibilidades. 0 s autores reforçaram a importância de produzir as oficinas como espaço clínico-político, permitindo que o sujeito estabeleça laços de cuidado consigo em vez da mera ocupação do tempo ${ }^{9}$.

Outros 19 artigos abordaram desafios referentes à efetivação das diretrizes da Reforma Psiquiátrica, apresentando as dificuldades envolvidas nas práticas alternativas, na criação de uma rede articulada de atenção e na falta de integralidade do atendimento. Cita-se como exemplo a experiência de Fortaleza (CE), que descreve estratégias de cuidado não articuladas aos princípios e às diretrizes do SUS, trazendo intervenções baseadas na prescrição medicamentosa e na internação ${ }^{10}$. Outro artigo revela a falta de articulação entre os serviços substitutivos e a APS, destacando que entre os principais desafios estão as questões relacionadas ao acolhimento dos sujeitos 
em sofrimento psíquico nos serviços da rede de saúde ${ }^{11}$.

Ainda no que se refere aos desafios, 11 artigos apontam a falta de cuidado interdisciplinar. Um deles relata uma experiência no Rio de Janeiro com práticas predominantemente baseadas no modelo biomédico de atenção à saúde, na psiquiatrização do cuidado em saúde mental, na burocratização do processo de trabalho e na centralização em ações intramuros. Nesse exemplo, a escuta e o acolhimento dos usuários se concentra na figura de um único profissional da saúde ${ }^{12}$.

Também há situações em que a discussão dos casos se realiza em equipe, exceto o médico, que permanece restrito a atendimentos individuais, como citado em um artigo, a partir de uma experiência em um Caps de Fortaleza ${ }^{13}$. Ao falar sobre a prática da equipe multiprofissional no serviço, revela-se que a categoria médica não se inclui no atendimento interdisciplinar, realizando apenas consultas individuais e demonstrando a prevalência do modelo biomédico com foco no diagnóstico e na doença em vez da escuta do sujeito ${ }^{13}$.

Contudo, nessa mesma experiência são descritas práticas que revelam articulação na rede de saúde, com profissionais que passaram a ampliar sua clínica com apoio matricial dos profissionais do serviço de referência - isto é, o Caps. Percebe-se uma tentativa de articular a proposta de matriciamento, de criar estratégias de capacitação para a APS, para que esta saiba lidar com as demandas de saúde mental por meio do trabalho em equipe. Contudo, ainda se mostra muito difícil e pontual a execução desse modelo proposto pelo SUS.

Em 4 artigos, os profissionais da saúde relatam sentir-se despreparados para atender o público no âmbito da saúde mental, corroborando a ideia de que acabam focalizando suas ações no cuidado do corpo em detrimento de questões subjetivas. Dessa forma, quando recebem pessoas em sofrimento psíquico, encaminham ao atendimento psicológico e/ou psiquiátrico, não demonstrando disponibilidade para o acolhimento, a escuta, a formação de vínculos e 0 apoio social. Por exemplo, 1 artigo indica 0 desconhecimento dos profissionais acerca do termo sofrimento psíquico, repercutindo uma prática distante dos princípios do SUS ao não considerar o conceito ampliado de saúde e de integralidade do atendimento ${ }^{14}$.

Percebe-se que os principais impasses relativos à atuação em serviços de APS, onde as práticas

\author{
...deve-se \\ possibilitar que o \\ usuário produza \\ vida para além dos \\ equipamentos $d a$ \\ saúde...
}

inovadoras constituem desafios e empecilhos para a equipe de saúde, corroboram o impedimento de tais práticas no cotidiano de trabalho. Ademais, assim como nos serviços de APS, há artigos relatando que a equipe do Caps, por vezes, também se vê limitada pela rotina do serviço, restringindo as ações do projeto terapêutico singular aos espaços institucionais. Isso implica o não reconhecimento das potencialidades dos territórios onde as pessoas vivenciam seus laços afetivos e de pertencimento com vistas ao cuidado em rede. No entanto, para efetivar a Reforma Psiquiátrica, deve-se possibilitar que 0 usuário produza vida para além dos equipamentos da saúde, de modo a estabelecer trocas simbólicas em seu território e exercitar sua cidadania.

Limitar as práticas aos espaços institucionais diverge da proposta de desinstitucionalização, o que se apresenta em pelo menos 6 artigos, nos quais as ações de acolhimento são descritas apenas no serviço e não na comunidade. Citamos o exemplo do Caps de João Pessoa (PB), que retrata a falta de interesse e de conhecimento teórico por parte da equipe da Estratégia Saúde da Família (ESF) para discutir os casos que demandam cuidado em saúde mental no território.

Dessa forma, os espaços comunitários e suas estratégias de produção de vida são, em sua maioria, invisibilizados ${ }^{15}$.

Outro achado desta pesquisa envolve o sofrimento psíquico dos cuidadores de usuários dos serviços de saúde mental: 5 artigos revelam a relevância de adotar políticas que também acolham os familiares e os profissionais desses serviços. Um dos artigos retrata a falta de acolhimento da família dos usuários, acarretando sua dificuldade de frequentar os serviços de saúde junto com o usuário por falta de apoio e escuta com foco no sofrimento ${ }^{16}$. Esse artigo reforça, ainda, a importância dos profissionais que atuam nos serviços de saúde mental buscarem a inclusão dos familiares nos projetos terapêuticos, integrando uma rede social; contudo, alerta-se que a inclusão não 
deve limitar-se à família, estendendo-se à comunidade em geral, o que contribui para a efetivação dos conceitos de integralidade do atendimento e de territorialidade.

Os profissionais da saúde consideram relevante 0 acolhimento com foco no sofrimento, que auxilie na promoção da saúde tanto dos usuários como dos cuidadores e dos próprios profissionais, revelando a necessidade de criar espaços para acolhimento e escuta de todos os envolvidos com vistas à redução do sofrimento psíquico no contexto do trabalho em serviços de saúde. Outro artigo traz uma experiência na qual as reuniões de equipe para discussão dos casos dos usuários dos serviços constituíram espaços importantes para acolher as angústias dos próprios profissionais da saúde, que, nessas situações, sentiam-se acolhidos e apoiados, encontrando suporte para o enfrentamento das situações de sofrimento ${ }^{17}$. Contudo, há outro artigo que revela 0 distanciamento nessa relação de cuidado com o profissional da saúde, denunciando, ainda, a precarização das condições de trabalho ${ }^{18}$. Nesse sentido, assim como nesse novo modo de cuidado com o sujeito em sofrimento há necessidade de assistência à família e de uma visão do sujeito inserido em seu território, também há necessidade de que a própria equipe de saúde possa ser cuidada.

A partir das considerações traçadas sobre os 29 artigos selecionados, pode-se dizer que este estudo apresenta dados relevantes para demonstrar que a Reforma Psiquiátrica ainda não se concretizou no Brasil, representando um desafio para todos os envolvidos. 0 cuidado com o chamado sujeito em crise não implica exclusivamente a assistência pontual por parte de um profissional da saúde, mas o trabalho em equipe multiprofissional para fortalecer a percepção das demandas em suas múltiplas dimensões ${ }^{19}$, devendo, ainda, articular a rede de cuidados e amparo ao sujeito dentro de seu território, a partir dos laços sociais e afetivos, sendo relevante o envolvimento de todos os profissionais da saúde nesse cuidado inclusive aqueles da categoria médica ${ }^{20,21}$.

\section{CONCLUSÃO}

Considerando os artigos identificados e selecionados, que relatam os desafios e impasses para efetivar ações cotidianas embasadas nas diretrizes propostas pelo SUS, as práticas revelam que o Brasil ainda carece de uma Reforma Psiquiátrica de facto.

\section{Deve-se considerar que a Reforma Psiquiátrica assume um amplo escopo na sociedade...}

As publicações apresentam os desafios inerentes à adoção de práticas diferentes no setor saúde, isto é, as dificuldades enfrentadas pelos profissionais em busca de novas formas de acolher os usuários dos serviços de saúde. Também se relata a não participação dos profissionais da categoria médica, ainda considerados no topo de uma pirâmide hierárquica, por deterem um saber mais especializado.

$\mathrm{Na}$ base dessa pirâmide se encontram profissionais mais numerosos de outras especialidades que trabalham de modo individualizado. Tal hierarquia de saberes rompe com a proposta de apoio e cogestão de Gastão Wagner, o chamado método paideia, também conhecido como método da roda, que visa a diminuir a fragmentação do trabalho em saúde, de modo a facilitar a promover 0 trabalho em equipe ${ }^{20}$. 0 s resultados e as discussões oferecidos por esta pesquisa ilustram que tal modelo de gestão ainda não se materializou no âmbito do SUS, pois em inúmeros serviços de saúde persiste o modelo hierárquico.

Deve-se considerar que a Reforma Psiquiátrica assume um amplo escopo na sociedade, no sentido de que também se trata de uma atualização jurídica, política, econômica e cultural. Quando se pensa a prática de um profissional da saúde para proporcionar suporte a um sujeito em crise, o foco recai sobre um aspecto de uma estrutura completa que precisa ser modificada para satisfazer as demandas desse sujeito em sofrimento psíquico.

Esta pesquisa envolve, ainda, as demandas de cuidado dos profissionais da saúde, o que implica, por exemplo, a necessidade de melhores condições de trabalho, de estabilidade no emprego, de concursos públicos e de planos de cargos e carreiras, para que esses profissionais se sintam pertencentes aos territórios onde trabalham e que não se sintam desmotivados diante das precárias condições de trabalho e da falta de reconhecimento profissional. Nesse sentido, a Reforma Psiquiátrica precisa se colocar como uma atualização que promove os laços trabalhistas, visto que a saúde do trabalhador afeta 
diretamente o cuidado oferecido pelo serviço a seus usuários.

Embora sejam notórios os avanços da Reforma Sanitária no Brasil, ainda há diversos impasses que precisam ser superados a fim de oferecer cuidado pautado em seus princípios aos usuários dos serviços de saúde que foram condenados por tanto tempo a maus tratos, isolamento e exclusão.

Indica-se a necessidade de continuação desta pesquisa, tendo em vista a relevância de aprofundar os conhecimentos acerca do cuidado e acolhimento às pessoas em sofrimento psíquico no âmbito da saúde mental brasileira.

\section{CONTRIBUIÇÃO DAS AUTORAS}

\author{
Karolyne Braga Moreira contribuiu com 0 \\ delineamento e a realização da pesquisa e a \\ estruturação e redação do manuscrito. Camilla Araújo \\ Lopes Vieira contribuiu com o delineamento da \\ pesquisa e a revisão crítica do manuscrito.
}

\section{REFERÊNCIAS}

1. Amarante P. Uma aventura no manicômio: a trajetória de Franco Basaglia. Hist Ciênc Saúde-Manguinhos [serial on the internet]. 1994 [cited 2017 0ct 11];1 (1):61-77. Available from: http://www.scielo.br/pdf/hcsm/v1n1/a06v01n1.pdf

2. Brasil. I Conferência Nacional de Saúde Mental: relatório final/8 ${ }^{a}$ Conferencia Nacional de Saúde [document on the internet]. Brasília (DF): Ministério da Saúde; 1988 [cited 2017 0ct 3]. Available from: http://bvsms.saude.gov.br/ bvs/publicacoes/0206cnsm_relat_final.pdf

3. Brasil. Relatório final da $2^{\text {a }}$ Conferência Nacional de Saúde Mental [document on the internet]. Brasília (DF): Ministério da Saúde; 1994 [cited 2017 Oct 3]. Available from: http://bvsms.saude.gov.br/bvs/publicacoes/2conf mental.pdf

4. Brasil. III Conferência Nacional de Saúde Mental: caderno informativo [document on the internet]. Brasília (DF): Ministério da Saúde; 2002 [cited 2018 May 7]. Available from: http://bvsms.saude.gov.br/bvs/ publicacoes/0210IIIcnsm.pdf

5. Brasil. Relatório Final da IV Conferência Nacional de Saúde Mental - Intersetorial [document on the internet]. Brasília (DF): Ministério da Saúde; 2010 [cited 2018 May 7]. Available from: http://conselho.saude.gov.br/biblioteca/ Relatorios/relatorio_final_IVcnsmi_cns.pdf

6. Merhy EE, Feuerwerker LCM. Novo olhar sobre as tecnologias de saúde: uma necessidade contemporânea. In: Mandarino ACS, Gomberg E, organizers. Leituras de novas tecnologias e saúde. São Cristóvão (SE): Ed. UFS; 2009. p. 29-74.
7. Cunha MV. Os periódicos em ciência da informação: uma análise bibliométrica. Ciência e Informação. 1985;14(1):3745.

8. Campos-Brustelo TN, Bravo FF, Santos MA. Contando e encantando histórias de vida em um centro de atenção psicossocial. SMAD, Rev Eletrônica Saúde Mental Alcool Drog [serial on the internet]. 2010 [cited 2017 0ct 18];6(1):111. Available from: http://pepsic.bvsalud.org/pdf/smad/ v6n1/07.pdf

9. Bittencourt IGS, Francisco DJ, Mercado LPL. Autoria em blog por pessoas em sofrimento psíquico: aprendizagem compartilhada, reconhecimento e promoção da saúde mental. Psicol Ciênc Prof [serial on the internet]. 2013 [cited 2017 0ct 18];33(4):988-99. Available from: http:// www.scielo.br/pdf/pcp/v33n4/v33n4a16.pdf

10. Lima DWC, Silveira LC, Vieira AN, Cunha BMC, Almeida ANS, Guerreiro EM. Referenciais teóricos que norteiam a prática de enfermagem em saúde mental. Esc Anna Nery Rev Enferm [serial on the internet]. 2014 [cited 2017 0ct 18];18(2):336-42. Available from: http://www.scielo.br/ pdf/ean/v18n2/1414-8145-ean-18-02-0336.pdf

11. Costa A, Silveira M, Vianna P, Silva-Kurimoto T. Desafios da atenção psicossocial na rede de cuidados do Sistema Único de Saúde do Brasil. Rev Port Enferm Saúde Mental [serial on the internet]. 2012 [cited 2017 0ct 18];7:46-53. Available from: http://www.scielo.mec.pt/pdf/rpesm/n7/ n7a08.pdf

12. Silveira DP, Vieira ALS. Saúde mental e atenção básica em saúde: análise de uma experiência no nível local. Ciênc Saúde Colet [serial on the internet]. 2009 [cited 2017 0ct 18];14(1):139-48. Available from: http://www.scielo.br/ $\mathrm{pdf} / \mathrm{csc} / \mathrm{v} 14 \mathrm{n} 1 / \mathrm{a} 19 \mathrm{v} 14 \mathrm{n} 1 . \mathrm{pdf}$

13. Andrade $A B, B o s i$ MLM. Qualidade do cuidado em dois centros de atenção psicossocial sob o olhar de usuários. Saúde Soc [serial on the internet]. 2015 [cited 2017 0ct 18];24(3):887-900. Available from: http://www.scielo.br/ pdf/sausoc/v24n3/0104-1290-sausoc-24-03-00887.pdf

14. Amarante AL, Lepre AS, Gomes JLD, Pereira AV, Dutra VFD. As estratégias dos enfermeiros para o cuidado em saúde mental no Programa Saúde da Família. Texto \& Contexto Enferm [serial on the internet]. 2011 [cited 2017 0ct 18];20(1):85-93. Available from: http://www.scielo.br/pdf/ tce/v20n1/10.pdf

15. Ferreira TPS. Produção do cuidado em saúde mental: desafios para além dos muros institucionais. Interface Comun Saúde Educ [serial on the internet]. 2017 [cited 2017 0ct 18];21(61):373-84. Available from: http://www. scielo.br/pdf/icse/v21n61/1807-5762icse-1807-576220160139.pdf

16. Covelo BSR, Badaró-Moreira MI. Laços entre família e serviços de saúde mental: a participação dos familiares no cuidado do sofrimento psíquico. Interface Comun Saúde Educ [serial on the internet]. 2015 [cited 2017 0ct 
18];19(55):1133-44. Available from: http://www.scielo.br/ pdf/icse/v19n55/1807-5762-icse-1807-576220140472.pdf

17. Lembo AP, Oliveira AP, Carrelli E. Conversando sobre desgaste mental no trabalho e suas possibilidades de enfrentamento: uma experiência no serviço público municipal de Guarulhos. Rev Bras Saúde 0cup [serial on the internet]. 2016 [cited 2017 0ct 18];41:e12. Available from: http://www.scielo.br/pdf/rbso/v41/2317-6369-rbso41-e12.pdf

18. Perez KV, Bottega CG, Merlo ARC. Análise das políticas de saúde do trabalhador e saúde mental: uma proposta de articulação. Saúde Debate [serial on the internet]. 2017 [cited 2017 0ct 18];41(Spec):287-98. Available from: http://www.scielo.br/pdf/sdeb/v41nspe2/0103-1104-sdeb41-spe2-0287.pdf

19. Goya N, Andrade LOM. Percepções de trabalhadores da saúde sobre práticas instituintes e desafios do Sistema Único de Saúde. Sanare (Sobral, Online) [serial on the internet]. 2015 [cited 2018 Apr 30];14(2):7-16. Available from: https://sanare.emnuvens.com.br/sanare/article/ view/824/495

20. Cunha GT, Campos GWS. Método paideia para co-gestão de coletivos organizados para o trabalho. Revista ORG \& DEMO [serial on the internet]. 2010 [cited 2018 May 7];11(1):31-46. Available from: http://www2.marilia.unesp. br/revistas/index.php/orgdemo/article/view/468/364

21. Brasil. Saúde mental no SUS: acesso ao tratamento e mudança do modelo de atenção. Brasília (DF): Ministério da Saúde; 2007.

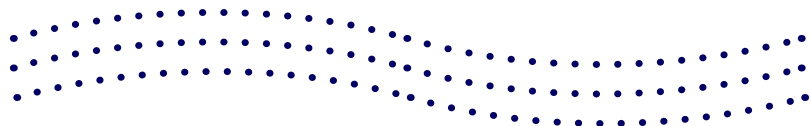

$\ldots \ldots \ldots \ldots \ldots \ldots \ldots$

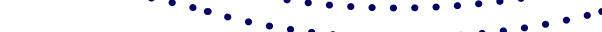

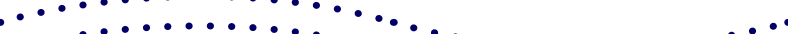

( $\ldots \ldots \ldots \ldots \ldots \ldots \ldots \ldots$

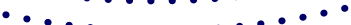
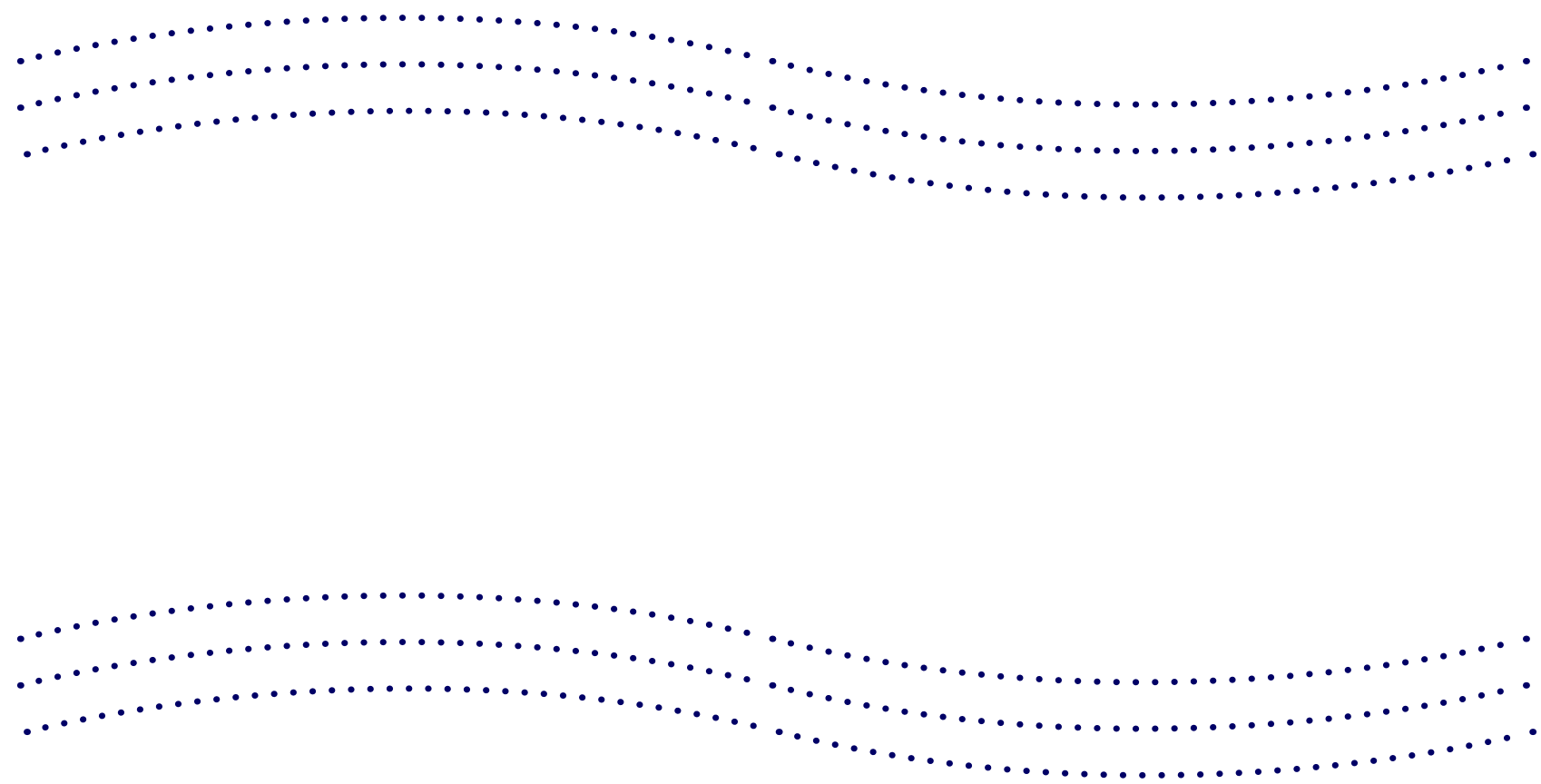